\title{
Development and implementation of AMOEBA-IL for classical and QM/MM simulations
}

G. Andrés Cisneros

Department of Chemistry
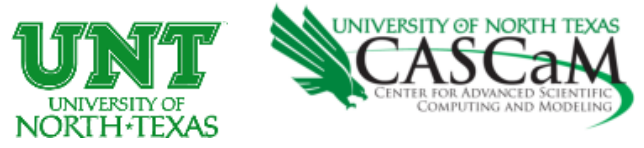

2020 ACS Spring Virtual National Meeting

QCisnerosRes; https://github.com/CisnerosResearch 


\section{Acknowledgements}

\section{Group Members}

Robert E. Duke

Sehr Naseem-Khan

Erik A. Vázquez-Montelongo

Mark A. Hix

Emmett Leddin

J. Enrique Vázquez-Cervantes

Madison Berger

Miguel Espinoza

Ally Smith

Krithika Ravishankar

Cory Shen

\section{Alumni}

Eric G. Kratz (Continental)

Hatice Gokcan (UNC)

Hedieh Torabifard (U. Mich)

Oleg Starovoytov (Southern U. LA)

Yi-Jung Tu (GA Tech)

Alice R. Walker (Stanford/SLAC)

\section{Collaborators}

Jean-Philip Piquemal (Sorbonne U.)

Pengyu Ren (UT Austin)

Thomas A. Darden (OpenEye)

Hugo Flores-Ruiz (U. de Guadalajara)

Andy Simmonett (NHLBI)

Computing Time

- CASCam

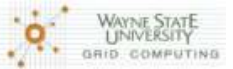

\section{XSEDE}

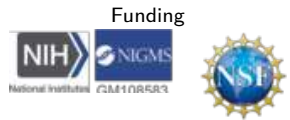

nVIDIA

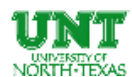




\section{Gaussian Electrostatic Model (GEM)}

Explicit representation of molecular electronic density 


\section{Gaussian Electrostatic Model (GEM)}

Explicit representation of molecular electronic density

Separate terms for intermolecular interactions, with energy/force errors $\leq 0.2 \mathrm{kcal} / \mathrm{mol}$ 


\section{Gaussian Electrostatic Model (GEM)}

Explicit representation of molecular electronic density

Separate terms for intermolecular interactions, with energy/force errors $\leq 0.2 \mathrm{kcal} / \mathrm{mol}$

$$
\tilde{\rho}=\sum_{t u v} c_{t u v} \Lambda_{t u v} ; \Lambda_{t u v}=(\alpha / \pi)^{3 / 2}(\partial / \partial x)^{t}(\partial / \partial y)^{u}(\partial / \partial z)^{v} \exp \left(-\alpha r^{2}\right)
$$

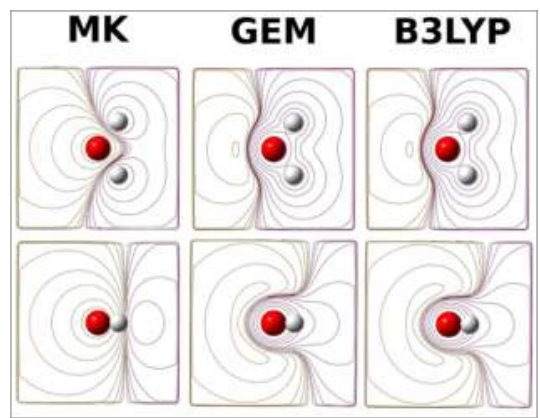

$\mathrm{H}_{2} \mathrm{O}$ Molecular Electrostatic potential (ESP) 


\section{Gaussian Electrostatic Model (GEM)}

Explicit representation of molecular electronic density

Separate terms for intermolecular interactions, with energy/force errors $\leq 0.2 \mathrm{kcal} / \mathrm{mol}$

$$
\tilde{\rho}=\sum_{t u v} c_{t u v} \Lambda_{t u v} ; \Lambda_{t u v}=(\alpha / \pi)^{3 / 2}(\partial / \partial x)^{t}(\partial / \partial y)^{u}(\partial / \partial z)^{v} \exp \left(-\alpha r^{2}\right)
$$

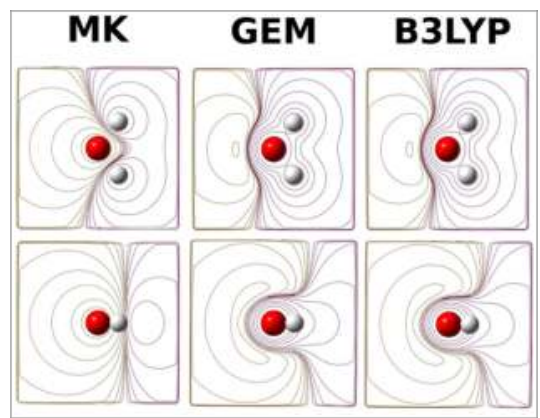

$\mathrm{H}_{2} \mathrm{O}$ Molecular Electrostatic potential (ESP)

Intrinsically finite multipoles $\rightarrow$ GEM-DM 


\section{GEM-Distributed Multipoles (GEM-DM)}

$q=h c_{000} \int \Lambda_{0} d_{r}=h c_{000}$

$\mu_{x}=h c_{100} \iiint x \Lambda_{100} d_{x} d_{y} d_{z}=h c_{100}$

$Q_{x^{2}}=h c_{200} \iiint x^{2} \Lambda_{200} d_{x} d_{y} d_{z}=2 h c_{200}$

$Q_{x y}=h c_{110} \iiint x y \Lambda_{110} d_{x} d_{y} d_{z}=2 h c_{110}$

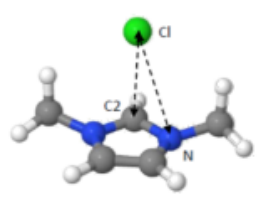

COULOMB ENERGY

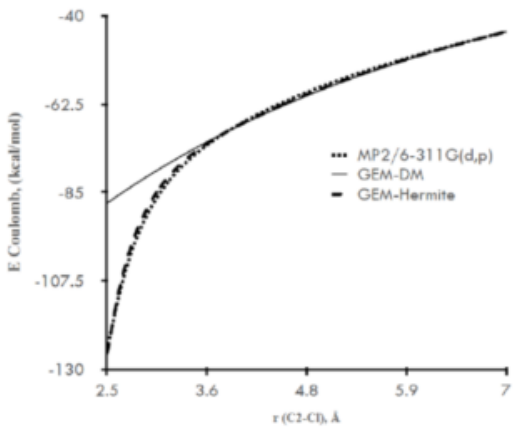




\section{Ionic Liquids}

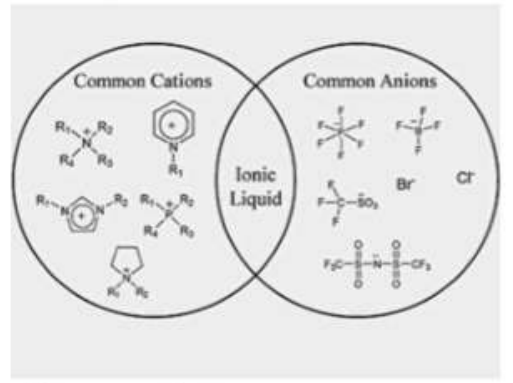

\section{Properties}

- High Viscosity

- Low vapor pressure

- Ion Conductive

- Water-immiscible

- Hygroscopic

Chem. Commun., 2012, 48, 7119-7130
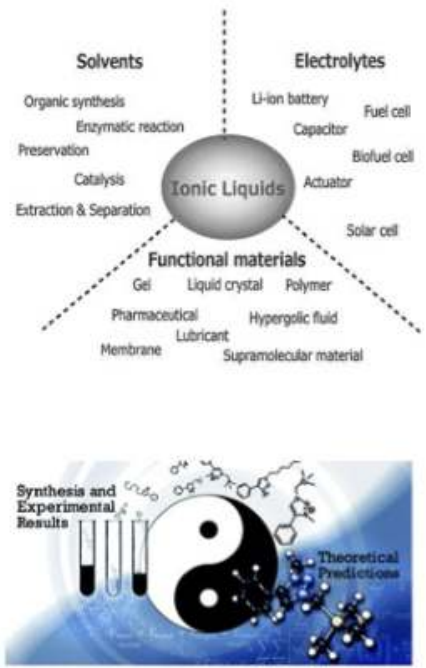


\section{AMOEBA-IL Parametrization}

- Use of high-level QM intermolecular interaction data (total and energy decomposition analysis) 


\section{AMOEBA-IL Parametrization}

- Use of high-level QM intermolecular interaction data (total and energy decomposition analysis)

Gaussian $\rightarrow$ Total intermolecular Energy as a QM reference RVS/SAPT $\rightarrow$ Individual Coulomb and Polarization energy

GEM-fit $\rightarrow$ Distributed multipoles from GEM Hermites TINKER $\rightarrow$ Bonded terms and vdW parameters-MM Energies 


\section{AMOEBA-IL Parametrization}

- Use of high-level QM intermolecular interaction data (total and energy decomposition analysis)

Gaussian $\rightarrow$ Total intermolecular Energy as a QM reference RVS/SAPT $\rightarrow$ Individual Coulomb and Polarization energy

GEM-fit $\rightarrow$ Distributed multipoles from GEM Hermites TINKER $\rightarrow$ Bonded terms and vdW parameters-MM Energies

$$
U_{v d W}=U_{T o t}(B S S E)-U_{C o u l}(E D A)-U_{P o l}(E D A)
$$




\section{Polarization Energy}

- Polarization

Redistribution of the electron

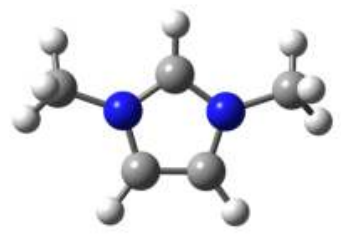
density in presence of an external electric field

$$
\begin{gathered}
\vec{\mu}_{i n d}=\alpha E \\
\vec{\mu}_{i n d}=\alpha\left(E_{\text {perm }}+E_{\text {ind }}\right) \\
U_{\text {pol }}=-0.5 \sum\left(\vec{\mu}_{i n d}\right) E
\end{gathered}
$$

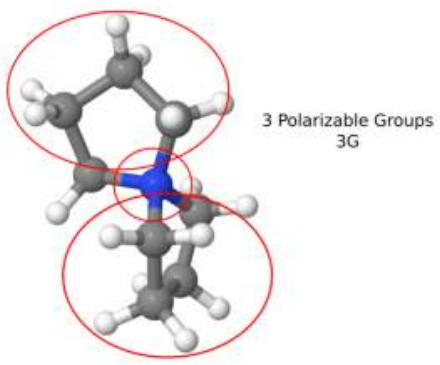




\section{Pyrrolidinium-based ionic liquids}
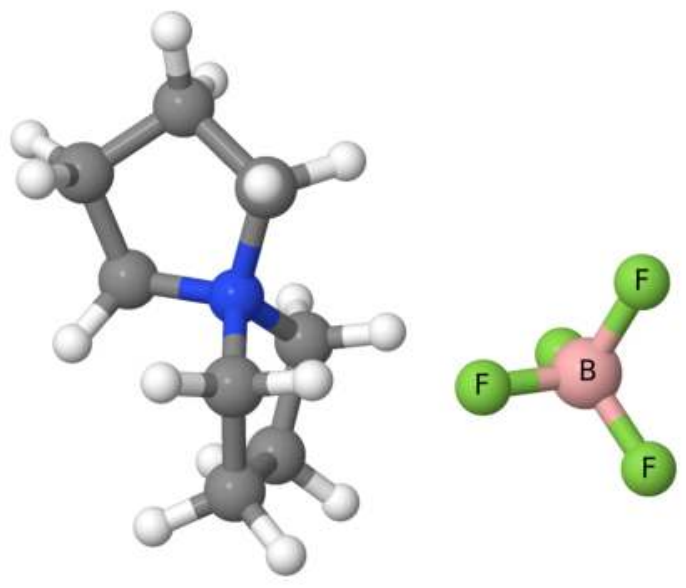


\section{$\left[\mathrm{sPyr}^{+}\right]\left[\mathrm{H}_{2} \mathrm{O}\right]$}

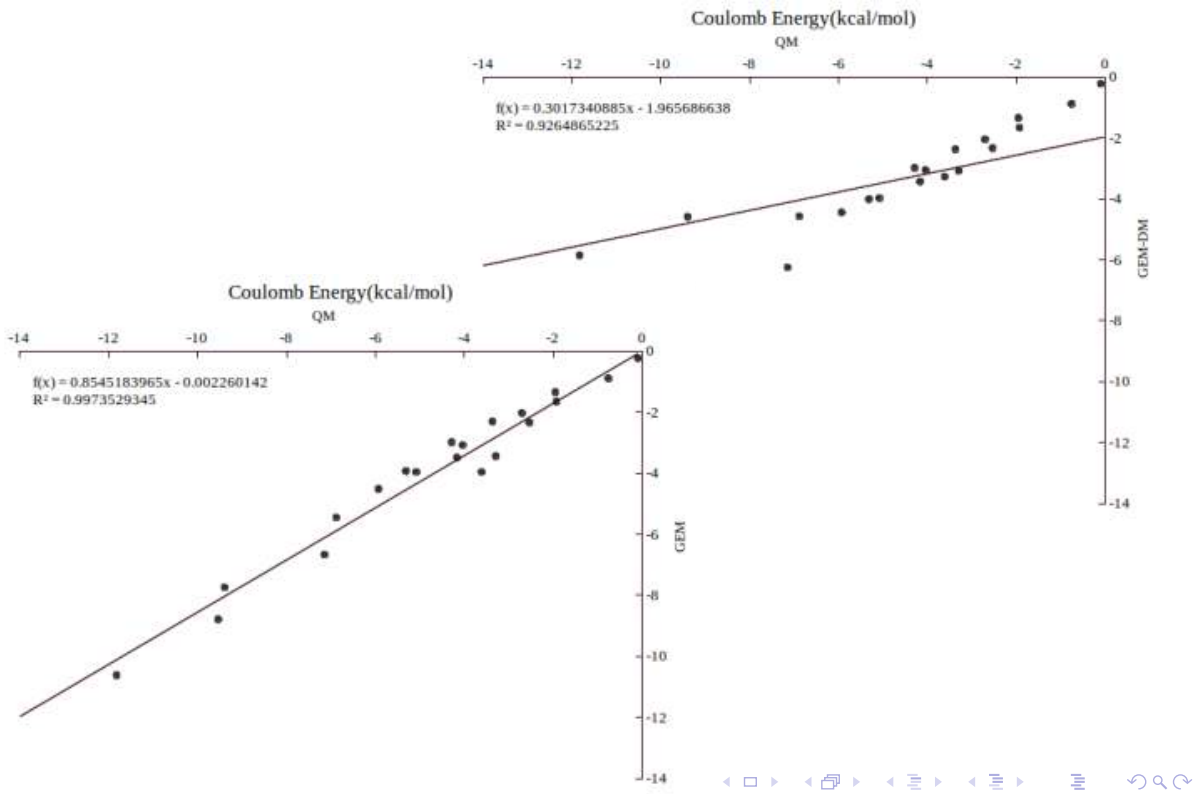




\section{$\left[\mathrm{sPyr}^{+}\right]\left[\mathrm{BF}_{4}^{-}\right]$}
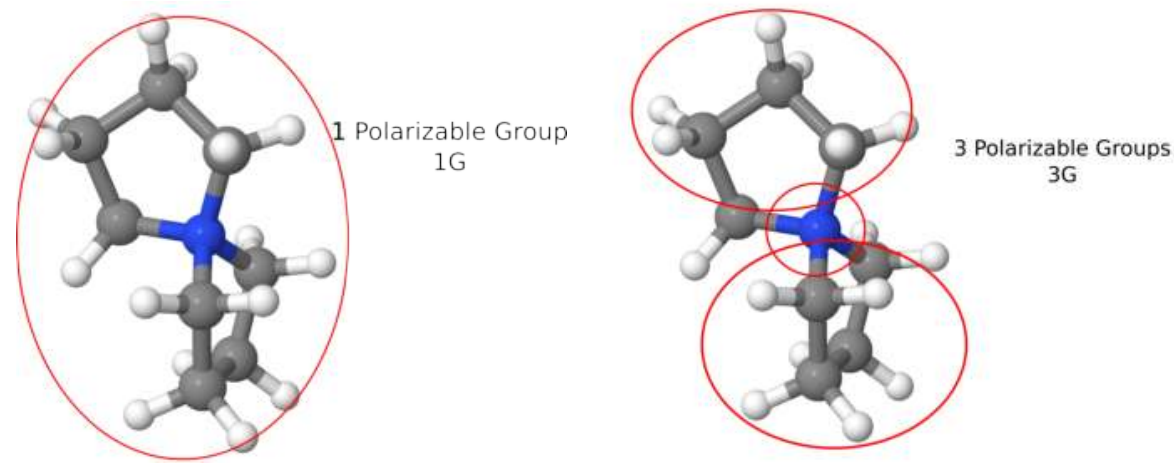


\section{$\left[\mathrm{sPyr}^{+}\right]\left[\mathrm{BF}_{4}^{-}\right]$Electrostatic Energy}
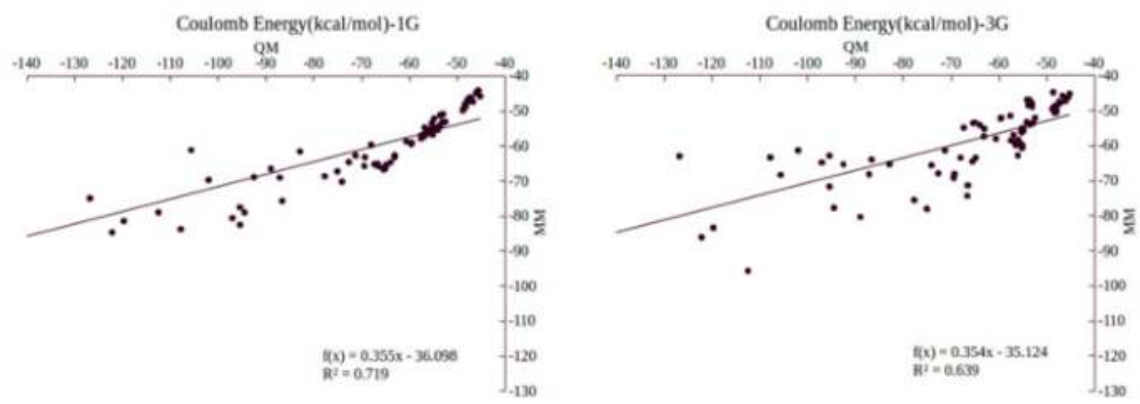


\section{$\left[\mathrm{sPyr}^{+}\right]\left[\mathrm{BF}_{4}^{-}\right]-$Total Energy}

Total Energy (kcal/mol)-1G

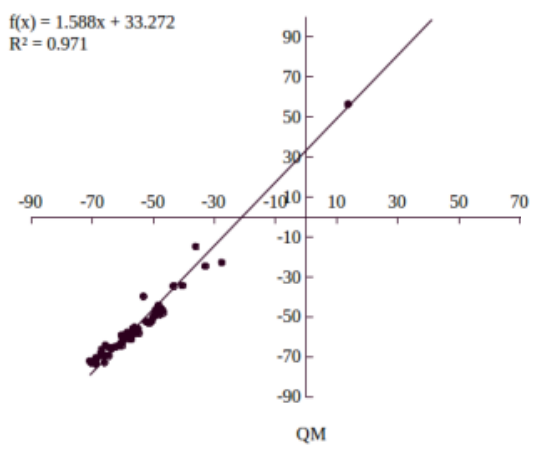

90

Total Energy (kcal/mol)-3G

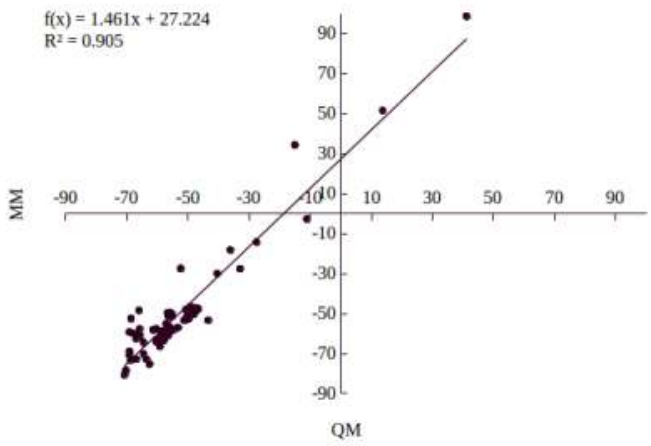

《口》《问 


\section{Simulation Details}

- gem.pmemd (in AMBER18/20) simulation package

- NPT ensemble ( $\mathrm{P}=1$ bar $)(\mathrm{T}=300-500 \mathrm{~K}) ; 216$ ion pairs; $10-20 \mathrm{~ns}$ production

- non-bonded cutoff $=8 \AA$, sPME, 1 fs timestep

- Berendsen thermostat/barostat 


\section{$\left[\mathrm{sPyr}^{+}\right]\left[\mathrm{BF}_{4}^{-}\right]$}
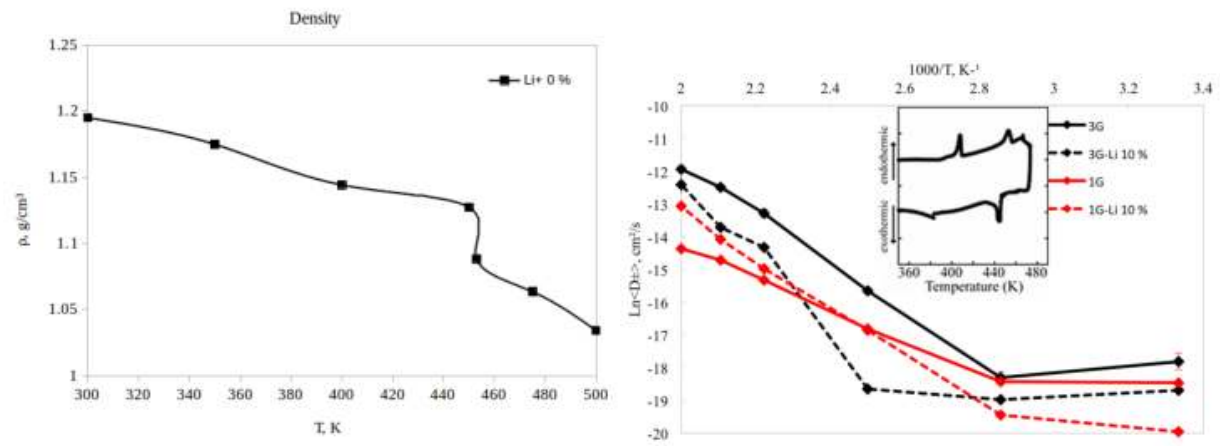


\section{$\left[\mathrm{sPyr}^{+}\right]\left[\mathrm{BF}_{4}^{-}\right]$}
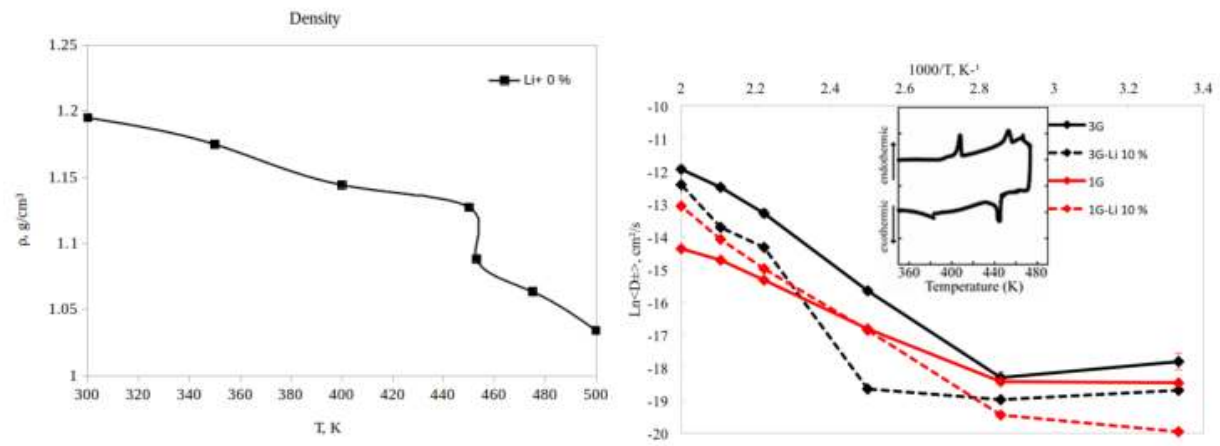

Experimental Melting Point: $448 \mathrm{~K}$ 


\section{Lanthanide Water Exchange in ILs}

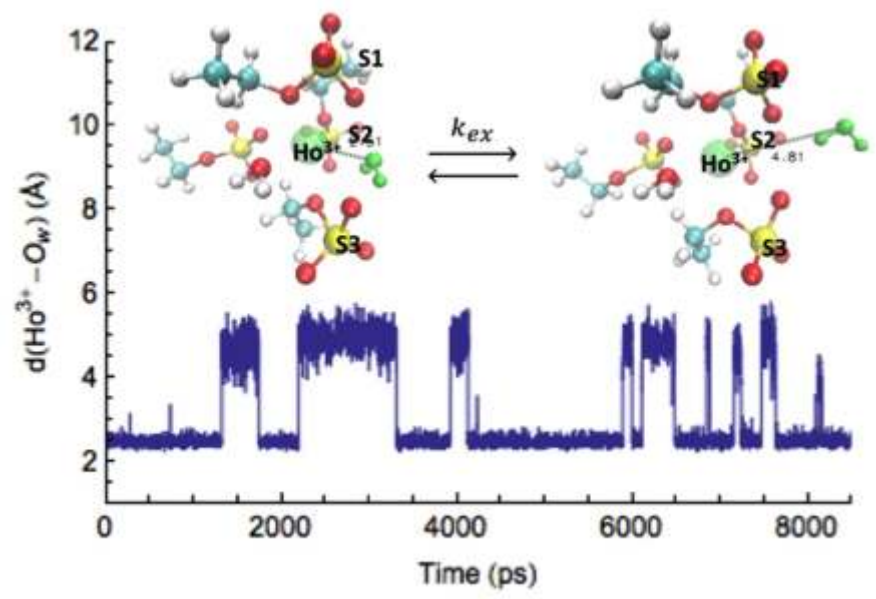




\section{Motivation}

- Understanding of dynamics of ligand exchange on Lanthanides is important for catalysis 


\section{Motivation}

- Understanding of dynamics of ligand exchange on Lanthanides is important for catalysis

- ${ }^{17} \mathrm{O}-\mathrm{NMR}$ Experiments have shown that $\mathrm{H}_{2} \mathrm{O}$ exchange rates of lanthinde ions in water is opposite to that in water/[EMIm] $\left[\mathrm{EtSO}_{4}\right]$ 


\section{Motivation}

- Understanding of dynamics of ligand exchange on Lanthanides is important for catalysis

- ${ }^{17} \mathrm{O}-\mathrm{NMR}$ Experiments have shown that $\mathrm{H}_{2} \mathrm{O}$ exchange rates of lanthinde ions in water is opposite to that in water/[EMIm] $\left[\mathrm{EtSO}_{4}\right]$

- Exchange mechanism is unknown 


\section{Motivation}

- Understanding of dynamics of ligand exchange on Lanthanides is important for catalysis

- ${ }^{17} \mathrm{O}-\mathrm{NMR}$ Experiments have shown that $\mathrm{H}_{2} \mathrm{O}$ exchange rates of lanthinde ions in water is opposite to that in water/[EMIm] $\left[\mathrm{EtSO}_{4}\right]$

- Exchange mechanism is unknown

- Use polarizable classical MD ... 


\section{Parametrization of Lanthanides}

Polarizabilities from Marjolin et al., CPL, 563, 25-29, 2013

\begin{tabular}{lllll}
\hline Metal ions & $\alpha\left(\AA^{3}\right)$ & $\mathrm{a}$ & $\mathrm{R}(\AA)$ & $\varepsilon(\mathrm{kcal} / \mathrm{m} \Omega \mathrm{l})$ \\
\hline $\mathrm{Gd}^{3 *}$ & $0.790^{*}$ & 0.146 & $3.30^{\mathrm{b}}$ & $8.5^{\mathrm{b}}$ \\
$\mathrm{Dy}^{3+}$ & $0.728^{*}$ & 0.140 & 3.23 & 15.0 \\
$\mathrm{Ho}^{3 *}$ & $0.702^{*}$ & 0.136 & 3.20 & 20.0 \\
\hline
\end{tabular}




\section{Parametrization of Lanthanides}

Polarizabilities from Marjolin et al., CPL, 563, 25-29, 2013

\begin{tabular}{lllll}
\hline Metal ions & $\alpha\left(\AA^{3}\right)$ & a & $\mathrm{R}(\AA)$ & $\varepsilon(\mathrm{kcal} / \mathrm{mol})$ \\
\hline $\mathrm{Gd}^{3 *}$ & $0.790^{*}$ & 0.146 & $3.30^{\mathrm{b}}$ & $8.5^{\mathrm{b}}$ \\
Dy $^{3+}$ & $0.728^{*}$ & 0.140 & 3.23 & 15.0 \\
$\mathrm{Ho}^{3 *}$ & $0.702^{*}$ & 0.136 & 3.20 & 20.0 \\
\hline
\end{tabular}

\begin{tabular}{|c|c|c|c|}
\hline Metal ions & & Current Work & Experiment \\
\hline \multirow[t]{2}{*}{$\mathrm{Gd}^{1 *}$} & $\mathrm{CN}$ & 8.8 & 8.7 \\
\hline & $\mathrm{Ln}-\mathrm{O}_{\mathrm{w}}$ & 2.38 & 2.42 \\
\hline \multirow[t]{2}{*}{$\mathrm{Dy}^{3+}$} & $\mathrm{CN}$ & 8.6 & 8.5 \\
\hline & $\mathrm{Ln}-\mathrm{Q}_{\mathrm{w}}$ & 2.36 & 2.38 \\
\hline \multirow[t]{2}{*}{$\mathrm{Ho}^{3 *}$} & $\mathrm{CN}$ & 8.7 & 8.4 \\
\hline & Ln-Qus. & 2.35 & 2.36 \\
\hline
\end{tabular}




\section{Simulation Details}

- gem.pmemd (AMBER18/20) simulation package

- NPT ensemble $(\mathrm{P}=1$ bar; $\mathrm{T}=300 \mathrm{~K}) ; 1$ lanthanide, $\mathrm{H}_{2} \mathrm{O}=511$; mixtures 83:150 $\mathrm{H}_{2} \mathrm{O}:\left[\mathrm{EtSO}_{4}\right]$ molar ratio $(1: 19 \mathrm{v} / \mathrm{v}) ; 99: 180 \mathrm{H}_{2} \mathrm{O}$ :[OTf] molar ratio $(1: 19 \mathrm{v} / \mathrm{v}) ; 10-20$ ns production

- non-bonded cutoff $=8 \AA$, sPME

- Berendsen thermostat/barostat 


\section{Exchange Rates}

\begin{tabular}{|cccc|}
\hline Solvent & Metal ion & $k_{\text {calc }}^{\text {exch }} \mathrm{s}^{-1}$ & $k_{\text {exp }}^{\text {exch }}, \mathrm{s}^{-1}$ \\
\hline $\mathrm{H}_{2} \mathrm{O}$ & $\mathrm{Gd}^{3+}$ & $1.30 \times 10^{9}$ & $8.30 \times 10^{8}$ \\
$\mathrm{H}_{2} \mathrm{O}$ & $\mathrm{Dy}^{3+}$ & $7.72 \times 10^{8}$ & $4.34 \times 10^{8}$ \\
$\mathrm{H}_{2} \mathrm{O}$ & $\mathrm{Ho}^{3+}$ & $4.75 \times 10^{8}$ & $2.14 \times 10^{8}$ \\
\hline $\mathrm{H}_{2} \mathrm{O} /\left[\mathrm{EMIm}^{3}\right]\left[\mathrm{EtSO}_{4}\right]$ & $\mathrm{Gd}^{3+}$ & $2.96 \times 10^{7}$ & $5.08 \times 10^{6}$ \\
$\mathrm{H}_{2} \mathrm{O} /\left[\mathrm{EMIm}^{6}\right]\left[\mathrm{EtSO}_{4}\right]$ & $\mathrm{Dy}^{3+}$ & $4.94 \times 10^{7}$ & $3.64 \times 10^{7}$ \\
$\mathrm{H}_{2} \mathrm{O} /\left[\mathrm{EMIm}^{3}\right]\left[\mathrm{EtSO}_{4}\right]$ & $\mathrm{Ho}^{3+}$ & $8.86 \times 10^{7}$ & $4.61 \times 10^{7}$ \\
\hline $\mathrm{H}_{2} \mathrm{O} /[\mathrm{EMIm}][\mathrm{OTf}]$ & $\mathrm{Gd}^{3+}$ & $1.30 \times 10^{8}$ & $1.3 \times 10^{7}$ \\
$\mathrm{H}_{2} \mathrm{O} /[\mathrm{EMIm}][\mathrm{OTf}]$ & $\mathrm{Dy}^{3+}$ & $2.00 \times 10^{8}$ & $1.4 \times 10^{8}$ \\
$\mathrm{H}_{2} \mathrm{O} /[\mathrm{EMIm}][\mathrm{OTf}]$ & $\mathrm{Ho}^{3+}$ & $2.60 \times 10^{8}$ & $1.5 \times 10^{8}$ \\
\hline
\end{tabular}

Tu Y.J., Allen M.A., GAC, PCCP, 18, 30323, 2016;

Tu Y.J., Lin Z., Allen M.A., GAC, JCP, 148, 0244503, 2018 


\section{Exchange Mechanisms in $\mathrm{H}_{2} \mathrm{O}$ and $[\mathrm{EMIm}]\left[\mathrm{EtSO}_{4}\right]$}
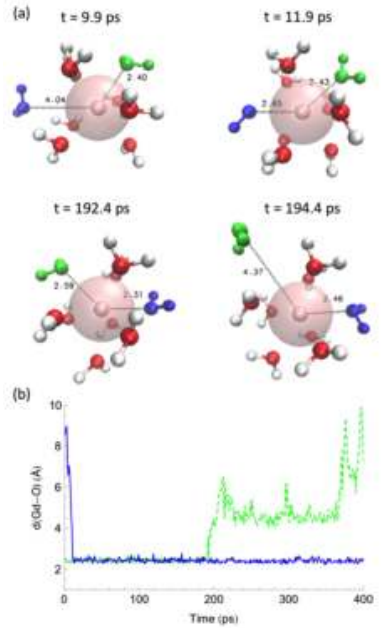


\section{Exchange Mechanisms in $\mathrm{H}_{2} \mathrm{O}$ and [EMIm] [EtSO $\left.{ }_{4}\right]$}
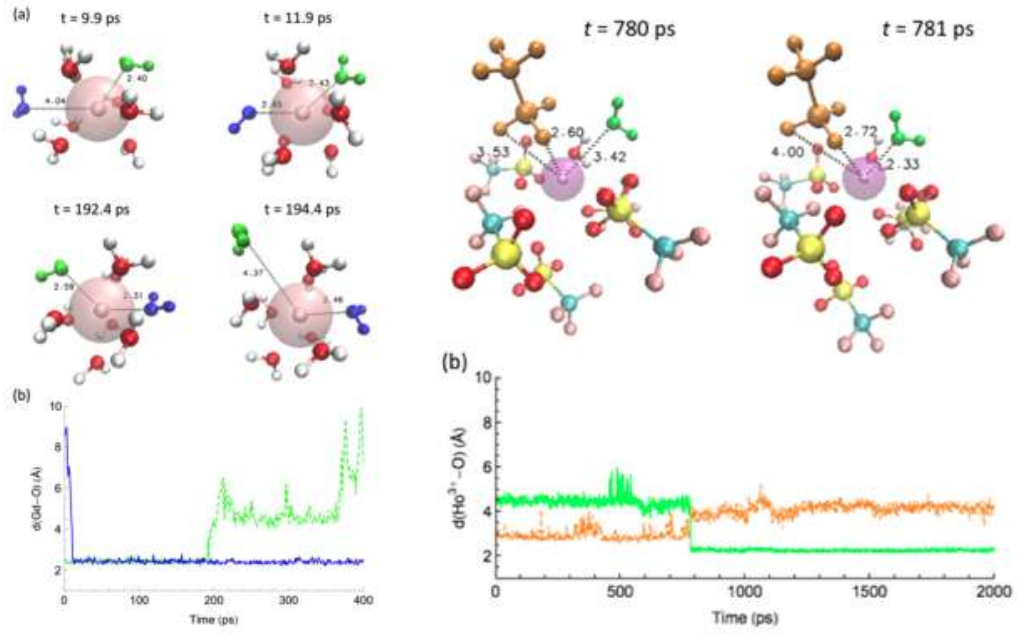

Tu Y.J., Allen M.A., GAC, PCCP, 18, 30323, 2016;

Tu Y.J., Lin Z., Allen M.A., GAC, JCP, 148, 0244503, 2018

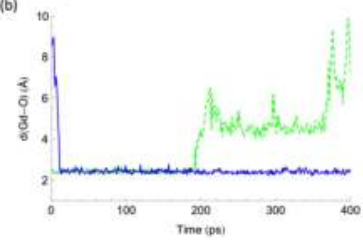




\section{Exchange Mechanisms in [EMIm][OTf]}
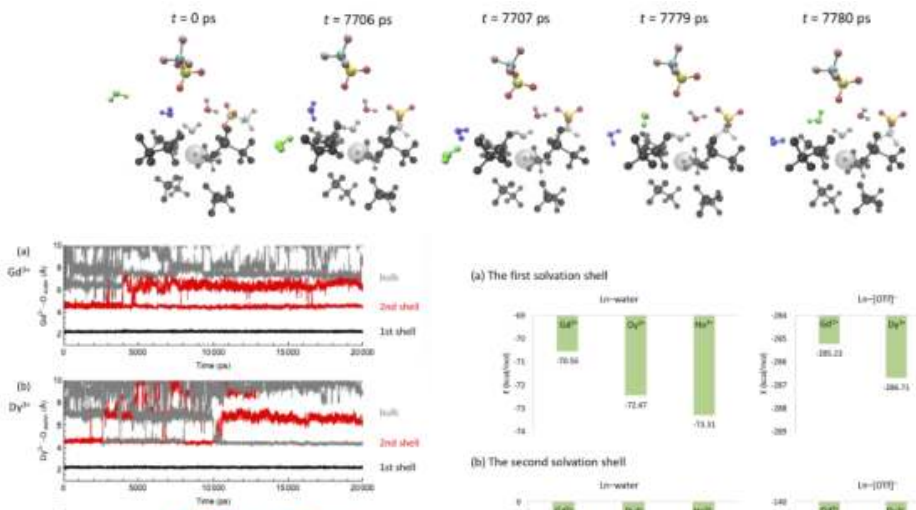

(a) The first solvation shelf

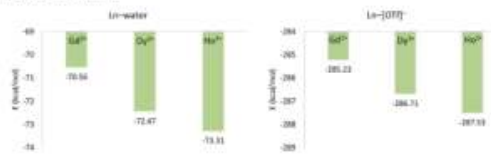

(b) The secand salvation sheil
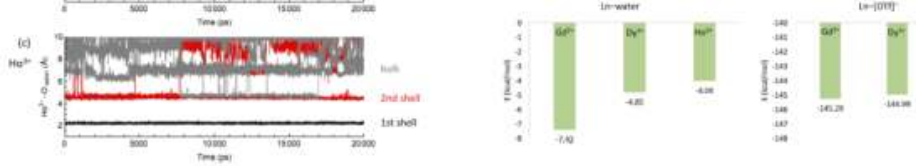

Tu Y.J., Allen M.A., GAC, PCCP, 18, 30323, 2016;

Tu Y.J., Lin Z., Allen M.A., GAC, JCP, 148, 0244503, 2018 


\section{Liquid-liquid extraction of Benzene}

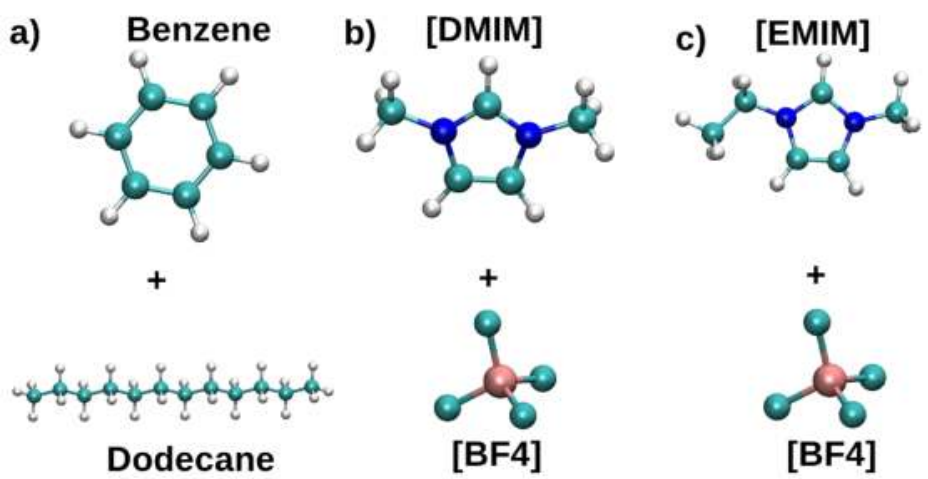

E.A. Vázquez Montelongo, GAC, H. Flores Ruiz, J. Mol. Liq. DOI: 10.1016/jmolliq.2019.111846 


\section{Simulation Details}

- MD with TINKER/OpenMM for AMOEBA

- Ternary systems: 500 molecules each (IL; benzene; dodecane), 300(150) ns production [EMIm] ([DMIm] $) x=y=4.5 \mathrm{~nm}, z=19.0 \mathrm{~nm}$

- Binary systems 500 molecules IL; 200 molecules solute (benzene or dodecane), 200 ns production

- NPT ensemble, $10 \AA$ cutoff for nonbonded, 2 fs timestep, 


\section{[DMIm][BF $\left.{ }_{4}\right]$ Density Profile}

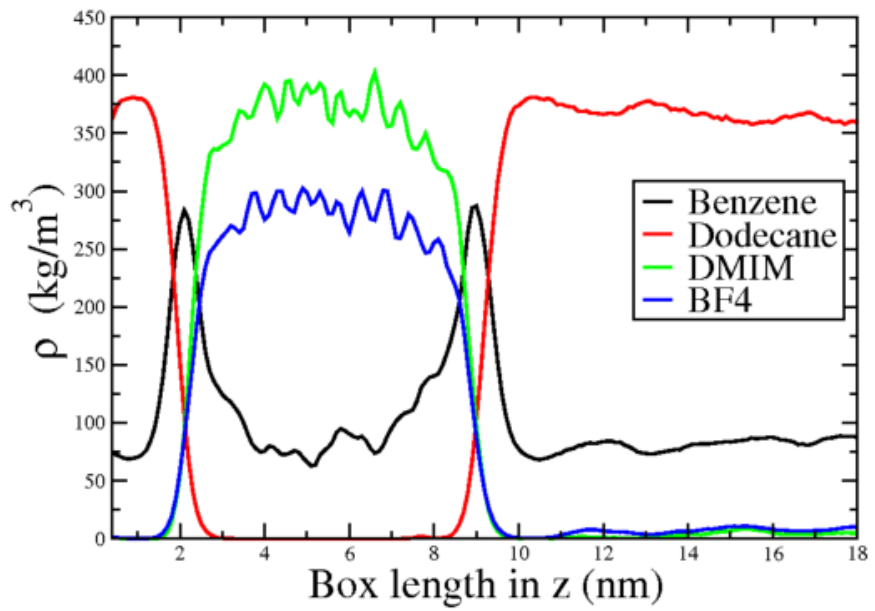

E.A. Vázquez Montelongo, GAC, H. Flores Ruiz, J. Mol. Liq. DOI: 10.1016/jmolliq.2019.111846 


\section{[EMIm][BF $\left.{ }_{4}\right]$ Density Profile}

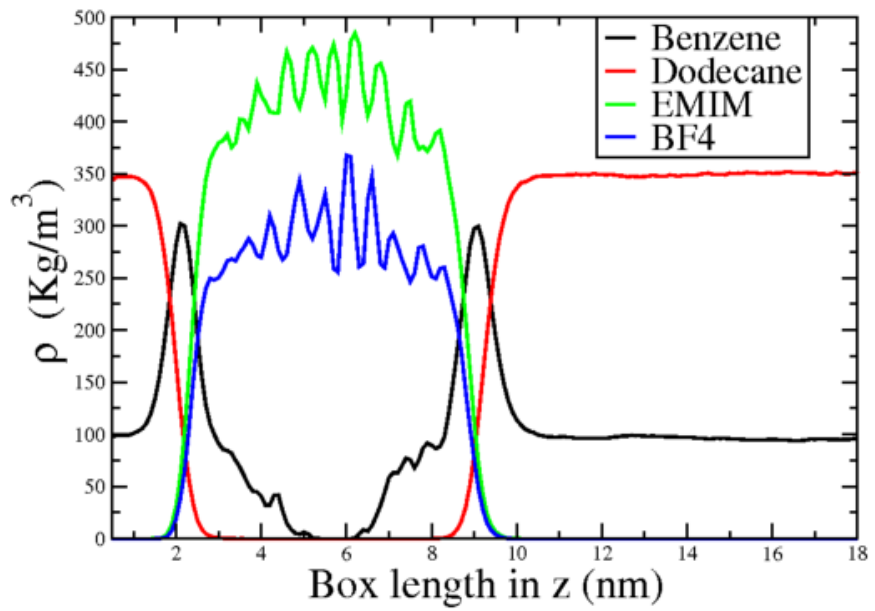

E.A. Vázquez Montelongo, GAC, H. Flores Ruiz, J. Mol. Liq. DOI: 10.1016/jmolliq.2019.111846 


\section{Cation SDF}

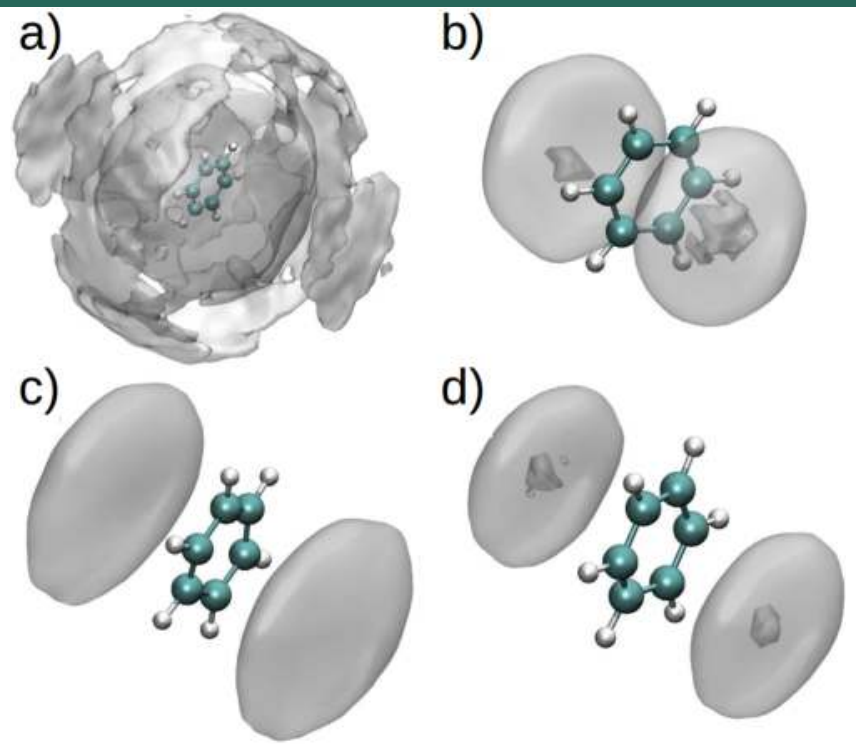

E.A. Vázquez Montelongo, GAC, H. Flores Ruiz, J. Mol. Liq. DOI: 10.1016/jmolliq.2019.111846 


\section{Anion SDF}
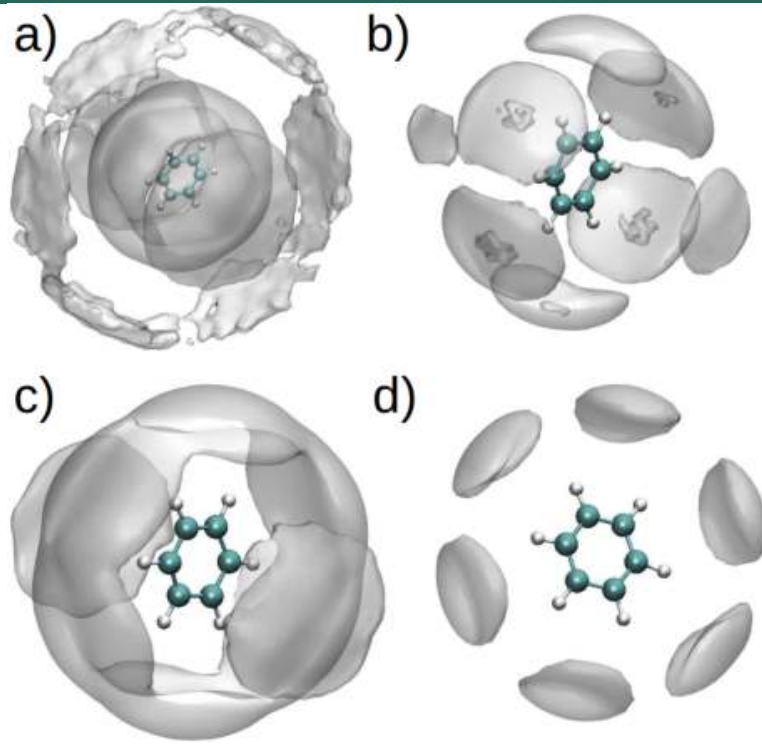

d)

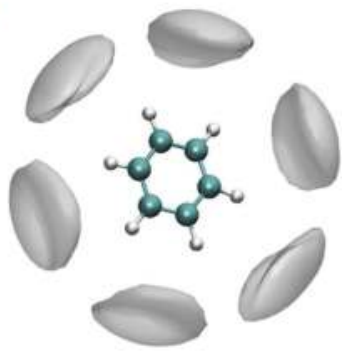

E.A. Vázquez-Montelongo, GAC, H. Flores Ruiz, J. Mol. Liq. DOI: 10.1016/jmolliq.2019.111846 


\section{LICHEM: Layered Interacting CHEmical Models}

\section{LCHEM: Layered Interacting CHEmical Models}
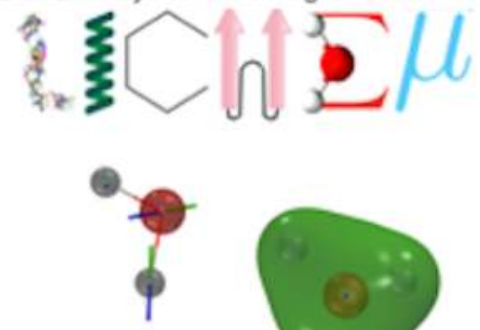

https://github.com/CisnerosResearch/LICHEM

Kratz E. et al., JCC, 37, 1019, 2016; Gokcan H. et al., JCTC, 15, 3056, 2019 


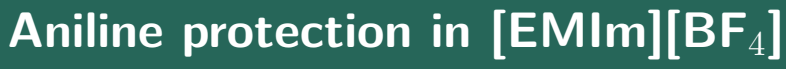

1)

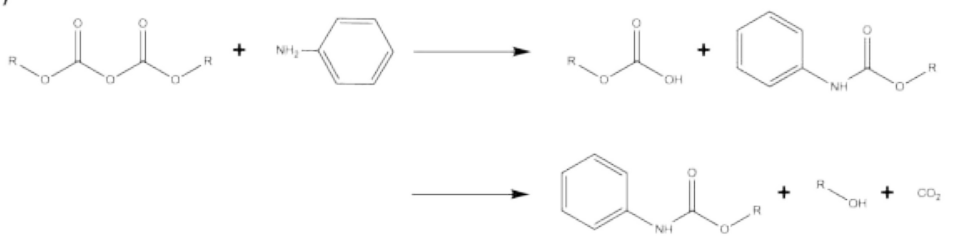

2)

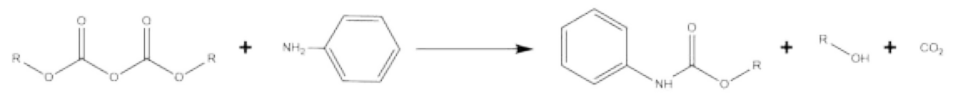

Vázquez-Montelongo et al., Molecules (2018), 23, 2830 


\section{Simulation Details}

- Initial MD with TINKER/OpenMM for AMOEBA, $7410 \mathrm{H}_{2} \mathrm{O}$, 189[EMIm] [BF 4 ], 1 aniline, 1 (Boc) ${ }_{2} \mathrm{O}$; NPT, 2 ns production

- QM/MM: 146 or $155 \mathrm{QM}$ atoms; $26688 \mathrm{MM}$ atoms; $\omega B 97 X-D / 6-31 G(d)$

- QSM path optimization; LREC/PME for long-range electrostatics

- $0.001 \AA$ RMSD, $0.005 \mathrm{au} /$ Bohr force RMS, $0.02 \mathrm{au} /$ Bohr max force 


\section{Importance of Polarization in QM/MM}

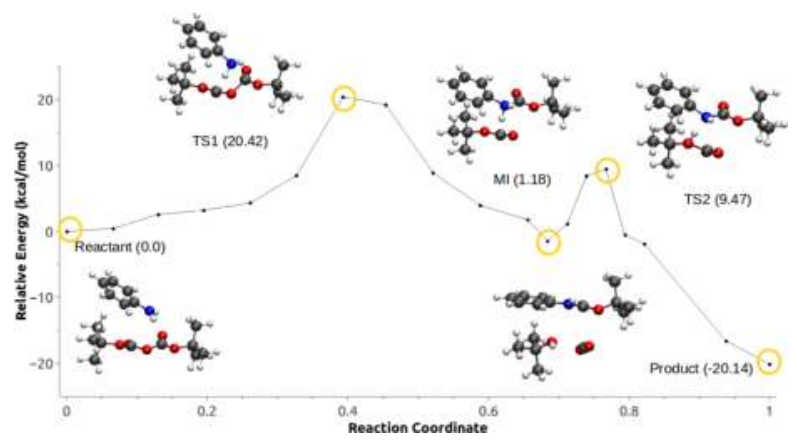




\section{Importance of Polarization in QM/MM}
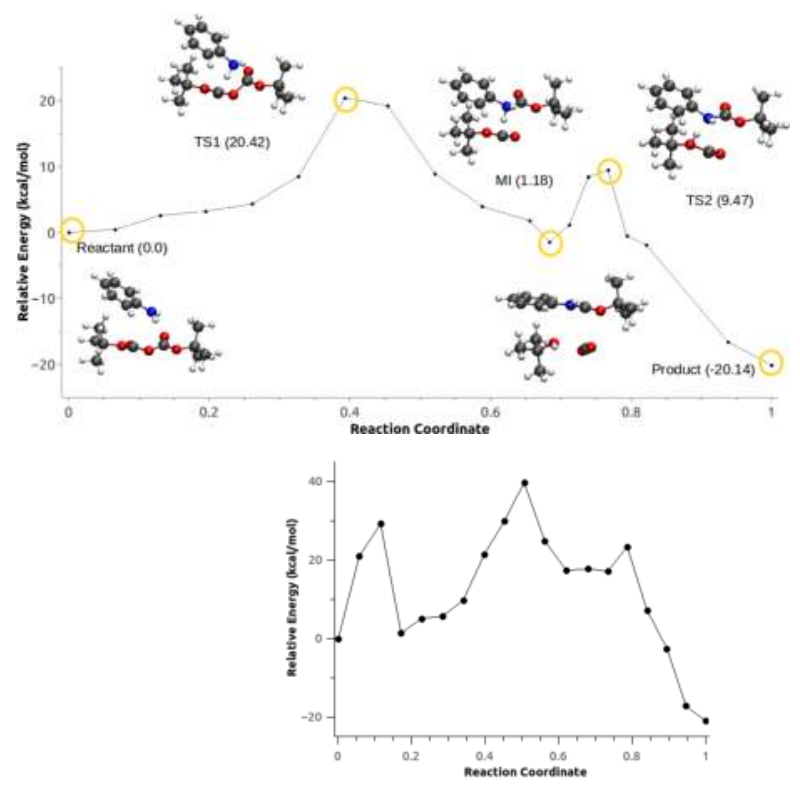


\section{Summary}

- gem.pmemd: MD platform for advanced FFs (experimental GEM implementation) 


\section{Summary}

- gem.pmemd: MD platform for advanced FFs (experimental GEM implementation)

- GEM distributed multipoles may be used in multipolar FF: AMOEBA-IL 


\section{Summary}

- gem.pmemd: MD platform for advanced FFs (experimental GEM implementation)

- GEM distributed multipoles may be used in multipolar FF: AMOEBA-IL

- "bottom-up" approach using ab initio data provides accurate parameters for AMOEBA-IL 


\section{Summary}

- gem.pmemd: MD platform for advanced FFs (experimental GEM implementation)

- GEM distributed multipoles may be used in multipolar FF: AMOEBA-IL

- "bottom-up" approach using ab initio data provides accurate parameters for AMOEBA-IL

- AMOEBA-IL predicts phase transition of [SPyr][4] at $453 \mathrm{~K}$, experimental melting pt. $=448 \mathrm{~K}$ 


\section{Summary}

- gem.pmemd: MD platform for advanced FFs (experimental GEM implementation)

- GEM distributed multipoles may be used in multipolar FF: AMOEBA-IL

- "bottom-up" approach using ab initio data provides accurate parameters for AMOEBA-IL

- AMOEBA-IL predicts phase transition of [SPyr] [4] at $453 \mathrm{~K}$, experimental melting pt. $=448 \mathrm{~K}$

- Water exchange rate trends on lanthanides reproduced by IL; MD shows exchange mechanism is associative in $\mathrm{H}_{2} \mathrm{O}$, and dissociative in $\mathrm{IL} / \mathrm{H}_{2} \mathrm{O}$ 


\section{Summary}

- gem.pmemd: MD platform for advanced FFs (experimental GEM implementation)

- GEM distributed multipoles may be used in multipolar FF: AMOEBA-IL

- "bottom-up" approach using ab initio data provides accurate parameters for AMOEBA-IL

- AMOEBA-IL predicts phase transition of [SPyr][4] at $453 \mathrm{~K}$, experimental melting pt. $=448 \mathrm{~K}$

- Water exchange rate trends on lanthanides reproduced by IL; MD shows exchange mechanism is associative in $\mathrm{H}_{2} \mathrm{O}$, and dissociative in $\mathrm{IL} / \mathrm{H}_{2} \mathrm{O}$

- $[\mathrm{DMIM}]\left[\mathrm{BF}_{4}\right]$ predicted to be a possible solvent for liquid-liquid extraction of benzene from gasoline 


\section{Summary}

- gem.pmemd: MD platform for advanced FFs (experimental GEM implementation)

- GEM distributed multipoles may be used in multipolar FF: AMOEBA-IL

- "bottom-up" approach using ab initio data provides accurate parameters for AMOEBA-IL

- AMOEBA-IL predicts phase transition of [SPyr][4] at $453 \mathrm{~K}$, experimental melting pt. $=448 \mathrm{~K}$

- Water exchange rate trends on lanthanides reproduced by IL; MD shows exchange mechanism is associative in $\mathrm{H}_{2} \mathrm{O}$, and dissociative in $\mathrm{IL} / \mathrm{H}_{2} \mathrm{O}$

- $[\mathrm{DMIM}]\left[\mathrm{BF}_{4}\right]$ predicted to be a possible solvent for liquid-liquid extraction of benzene from gasoline

- LICHEM enables combination of unmodified QM and MM codes 


\section{Summary}

- gem.pmemd: MD platform for advanced FFs (experimental GEM implementation)

- GEM distributed multipoles may be used in multipolar FF: AMOEBA-IL

- "bottom-up" approach using ab initio data provides accurate parameters for AMOEBA-IL

- AMOEBA-IL predicts phase transition of [SPyr][4] at $453 \mathrm{~K}$, experimental melting pt. $=448 \mathrm{~K}$

- Water exchange rate trends on lanthanides reproduced by IL; MD shows exchange mechanism is associative in $\mathrm{H}_{2} \mathrm{O}$, and dissociative in $\mathrm{IL} / \mathrm{H}_{2} \mathrm{O}$

- $[\mathrm{DMIM}]\left[\mathrm{BF}_{4}\right]$ predicted to be a possible solvent for liquid-liquid extraction of benzene from gasoline

- LICHEM enables combination of unmodified QM and MM codes

- Polarization effects are important for $\mathrm{QM} / \mathrm{MM}$ reactivity calculations in highly charged media 


\section{Acknowledgements}

\section{Group Members}

Robert E. Duke

Sehr Naseem-Khan

Erik A. Vázquez-Montelongo

Mark A. Hix

Emmett Leddin

J. Enrique Vázquez-Cervantes

Madison Berger

Miguel Espinoza

Ally Smith

Krithika Ravishankar

Cory Shen

\section{Alumni}

Eric G. Kratz (Continental)

Hatice Gokcan (UNC)

Hedieh Torabifard (U. Mich)

Oleg Starovoytov (Southern U. LA)

Yi-Jung Tu (GA Tech)

Alice R. Walker (Stanford/SLAC)

\section{Collaborators}

Jean-Philip Piquemal (Sorbonne U.)

Pengyu Ren (UT Austin)

Thomas A. Darden (OpenEye)

Hugo Flores-Ruiz (U. de Guadalajara)

Andy Simmonett (NHLBI)

Computing Time

- CASCam

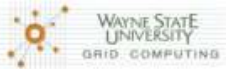

\section{XSEDE}

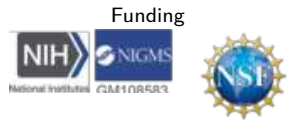

nVIDIA

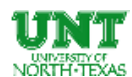

